\title{
Body Information Analysis based Personal Exercise Management System
}

\author{
Jongwon Lee, Hyunju Lee, Donggyun Yu, and Hoekyung Jung \\ Department of Computer Engineering, PaiChai University, Doma2-Dong, SeoGu, DaeJeon, Korea
}

\begin{tabular}{|c|c|}
\hline Article Info & ABSTRACT \\
\hline Article history: & Recently, people's interest in health is deepening. So health-related systems \\
\hline Received Oct 29, 2017 & $\begin{array}{l}\text { are being developed. Existing exercise management systems provided users } \\
\text { with exercise related information using PC or smart phone. However, there is }\end{array}$ \\
\hline Revised Feb 20, 2018 & a problem that the accuracy of the algorithm for analyzing the user's body \\
\hline Accepted Mar 14, 2018 & information and providing information is low.In this paper, we analyze users' \\
\hline Keyword: & $\begin{array}{l}\text { system that provides the user with necessary information through } \\
\text { recommendation algorithm. It informs the user of exercise intensity and }\end{array}$ \\
\hline BMI & momentum, and graphs the exercise history of the user. It also allows the \\
\hline BMR & user to refer to the fitness history of other users in the same BMI group. This \\
\hline Recommendation & allows the user to receive more personalized services than the existing \\
\hline U-healthcare & exercise management system, thereby enabling efficient exercise. \\
\hline User analysis & $\begin{array}{r}\text { Copyright }\left(\begin{array}{l}2018 \text { Institute of Advanced Engineering and Science. } \\
\text { All rights reserved. }\end{array}\right. \\
\text {. }\end{array}$ \\
\hline \multicolumn{2}{|l|}{ Corresponding Author: } \\
\hline \multicolumn{2}{|c|}{$\begin{array}{l}\text { Hoekyung Jung, } \\
\text { Departement of Computer Engineering, } \\
\text { Paichai University, } \\
\text { 155-40 University Road, Seogu, DaeJeon, Korea. } \\
\text { Email: hkjung@pcu.ac.kr }\end{array}$} \\
\hline
\end{tabular}

\section{INTRODUCTION}

As the number of consumers for a healthy life increases, a system for periodically checking physical condition and health using a smart phone or a wearable device is being developed. By using this, the past and present state of the user can be known and the service [1-3]. However, since existing systems record the user's movements and provide information accordingly, there is a problem that they cannot help users who have not understanding of motion [4-8]. In order to solve this problem, it is required to develop a system for analyzing the user's body information and providing a service accordingly.

In this paper, we analyze user BMI index and BMR value and provide service. The service provided by the proposed system analyzes the BMI index then recommends exercise intensity. Next analyze BMR values and recommend momentum. It also provides a graphical representation of the records of used exercise equipment.

System recommend to user exercise intensity and momentum can select the exercise device and can view the calories consumed in real time and select whether to transmit the exercise amount to the server. Also, records of other users belonging to the same BMI group as the user can be referred to This allows the user to compare other user's select the exercise equipment. Using the proposed system, the user can get more information and exercise more efficiently than existing exercise management system. And user can efficient planning.

\section{DESIGN}

This chapter describes the design of the proposed system. 


\subsection{Design of System}

Figure 1 shows the structure of the system and Figure 2 shows the flow chart of the system.

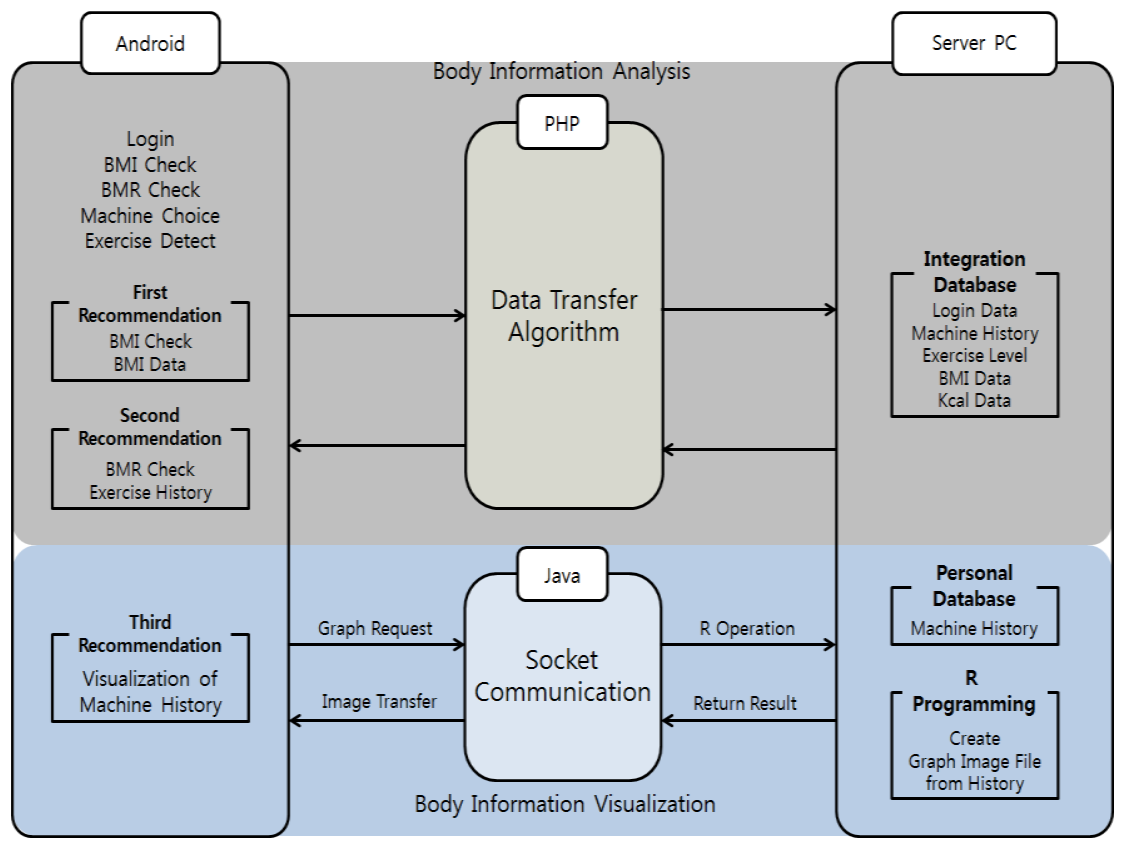

Figure 1. System Configuration

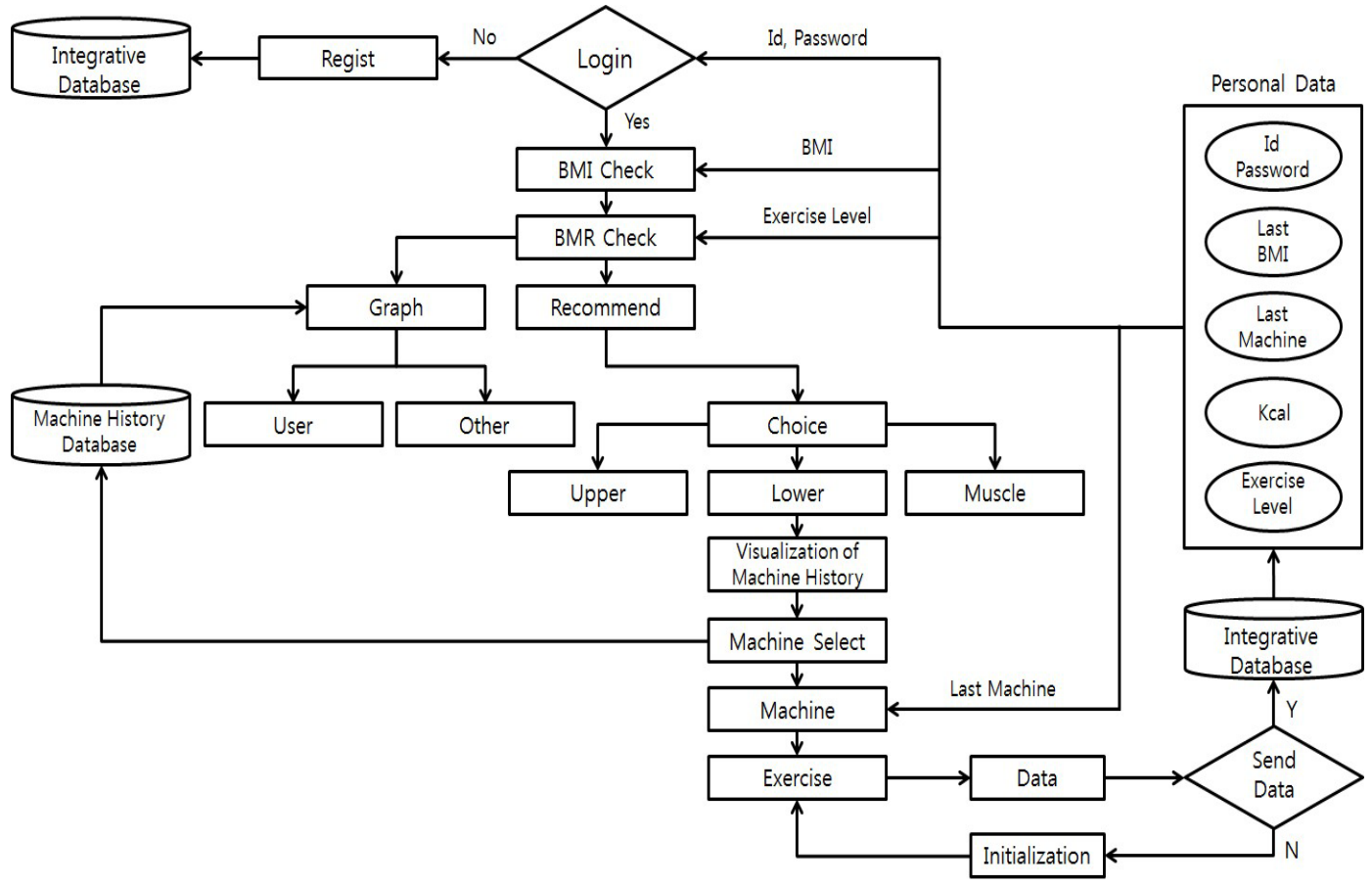

Figure 2. System Flow Char

The structure of the system consists of Android, PHP, Java, and server PC. Android, PHP and server $\mathrm{PC}$ is interlocked for analyzing the user's body information. The user will be able to enter and check the BMI index and BMR value in the Android application and be informed accordingly. The user's information is 
transferred to the database and in the integrated database, user information is analyzed and grouped according to BMI index. For visualizing user information, Android, Java, and server PC communicate. We store the exercise equipment used by the user in a personal database, construct a history and analyze it through $\mathrm{R}$ programming. The analyzed data is sent to Android via Java socket communication and displayed as a graph. Also, showing the used exercise equipment by users belonging to the same BMI group. So user can compare other users exercise history.

He system beginning is to the application run on the Android smartphone. The main functions of the application are login, BMI index check, BMR value check, fitness history reference, exercise equipment selection, motion detection function. A user who has not been registered yet should fill in information such as name, sex, age, ID, password, and momentum for membership registration. Based on the log-in and BMI index confirmation, the recommended exercise intensity is recommended by the first recommendation algorithm, and the second recommendation algorithm is based on the BMR value confirmation. It also graphically displays the exercise equipment that the user has already used, and also graphs the exercise equipment records used by other users belonging to the same BMI group as the user. Based on two recommendation algorithms and exercise history reference, the user is provided with appropriate information for the body. Next, when the user selects the exercise device the exercise device selected as the personal database is stored. The third recommendation algorithm is provides information on commonly used exercise equipment. Also when the user performs the exercise, it displays the consumed calorie value in real time. After the exercise, the user transfers the exercise amount to the server or initializes it according to the user's selection.

\subsection{Recommendation Algorithm}

The proposed system consists of three recommended algorithms. The first recommendation algorithm recommends exercise intensity, the second recommendation algorithm recommends the momentum, and the third recommendation algorithm recommends a exercise mahcine.

Figure 3 is a flow chart of the first recommendation algorithm of the fitness system of the proposed system.

The first recommendation algorithm calculates the BMI index of the user. We recommend exercise intensity to the user according to the BMI index. The exercise intensity in the application is expressed as Level. BMI groups will also be divided and recommended for exercise intensity. The correlation between the BMI index and exercise intensity was set based on information in the U-Healthcare field.

Figure 4 is a flowchart of the second recommendation algorithm.

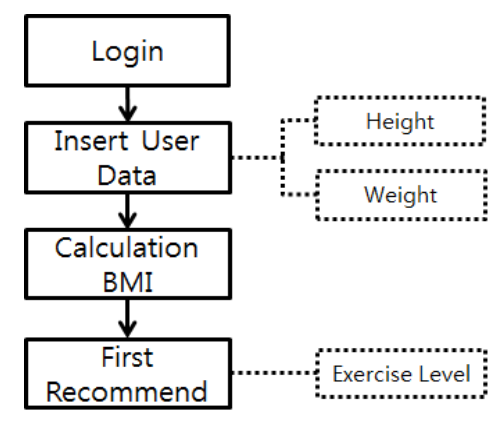

Figure 3. First Recommendation Algorithm

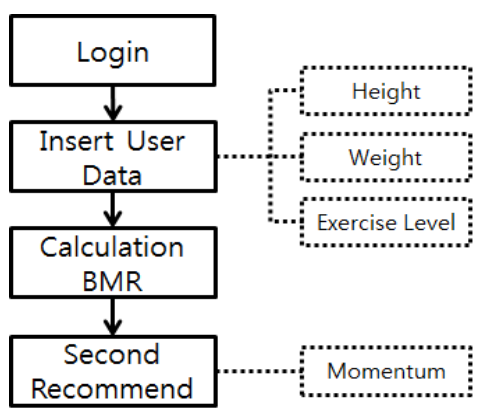

Figure 4. Second Recommendation Algorithm

The second recommendation algorithm recommends the amount of exercise that needs to be carried out in one day, taking into account the BMR of the user and the level of exercise the user has entered. The using Harris-Benedict Equation for BMR value calculates and recommends momentum to the user.

Figure 5 is a flowchart of the third recommendation algorithm.

The third recommendation algorithm provides information based on the data of the user's BMI group. When the user selects the exercise machine, the Toast message is displayed to the exercise devices used by other users belonging to the same BMI group. Using the information provided by the application, the user can exercise more efficiently than the services provided by the existing exercise management systems or plan the exercise. It also shows the calories consumed by the user in real time. And it shows the effect of the exercise machine to the user by specifying the improved muscle area when the exercise is performed using the corresponding exercise machine. 


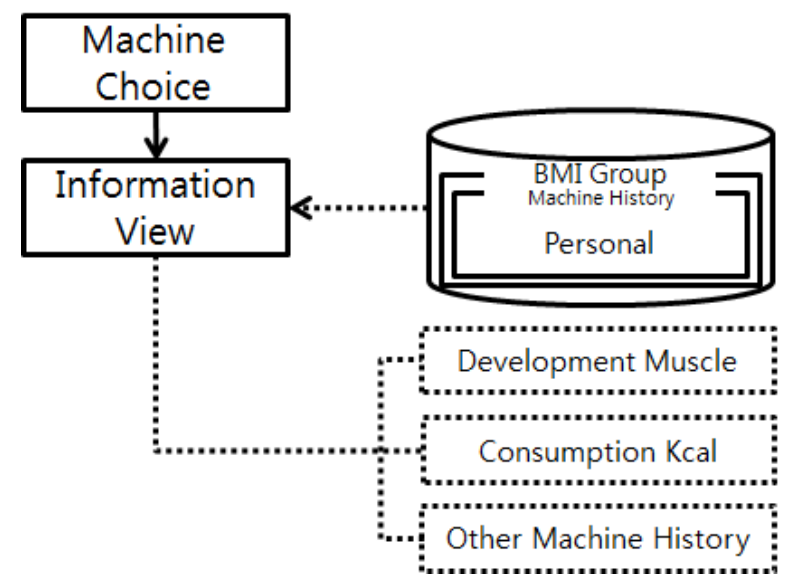

Figure 5. Third Recommendation Algorithm

\section{IMPLEMENTATION AND REVIEW}

\subsection{Implementation Environment}

The operating systems is Windows 7, CPU i5-4690, and RAM 8G. The smartphones used are Galaxy S5 and Galaxy S6 Edge. The tool used Eclipse to control the smartphone and the server, and APM Setup to connect the PC and the smartphone.

\subsection{Implementation}

Figure 6 shows the screen for analyzing the user's body information.

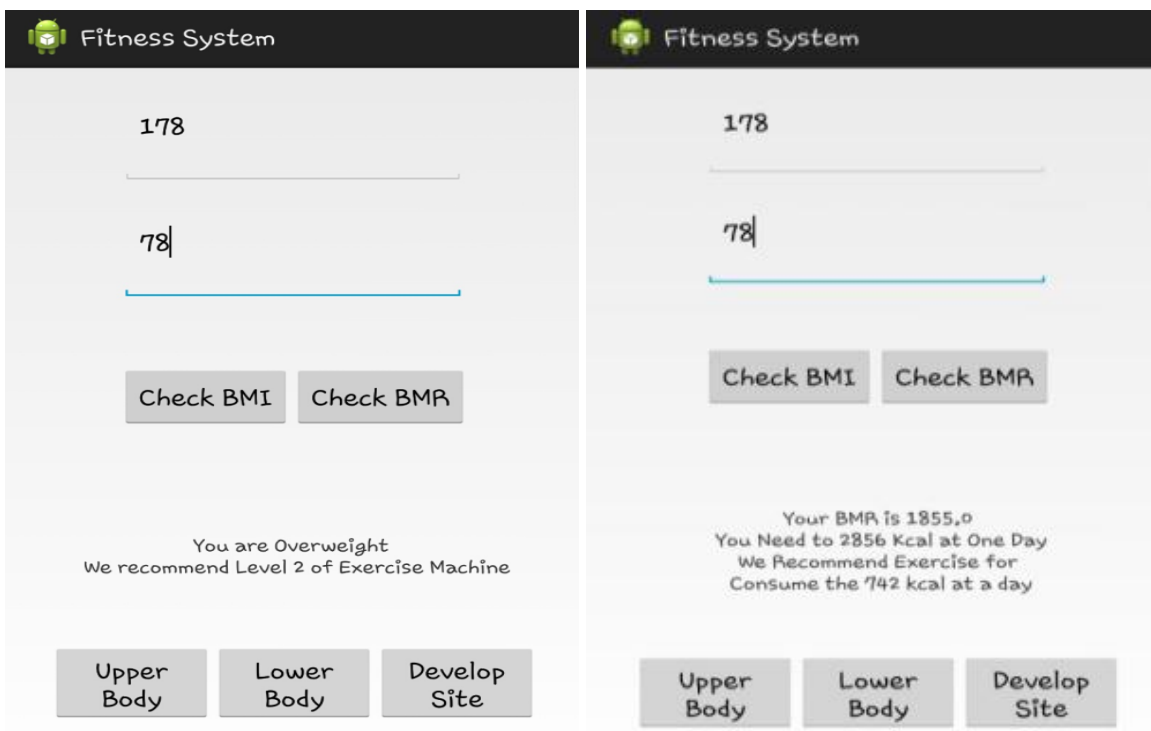

Figure 6. Analysis User Body Information

Figure 6 analyzes the height and weight entered by the user and recommends exercise intensity to the user using the Check BMI button. Also, when you press the Check BMR button, you will be informed of the user's metabolism and recommended exercise amount.

Figure 7 is a graphical representation of the exercise history. It is also the records of the users belonging to the same BMI group as the user. The user can review and modify the plan with respect to the exercise by referring to the record of the exercise equipment he or she has used and the records of other users. 


\section{윈 Fitness System}

윕 Fitness System

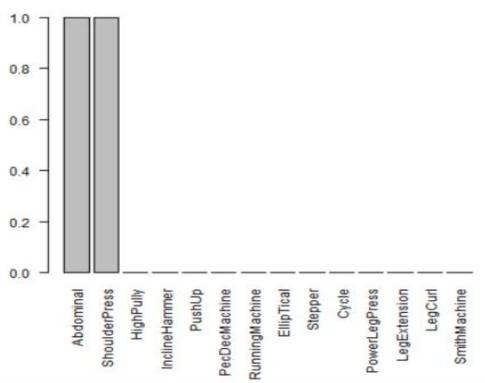

Group Graph Personal Graph

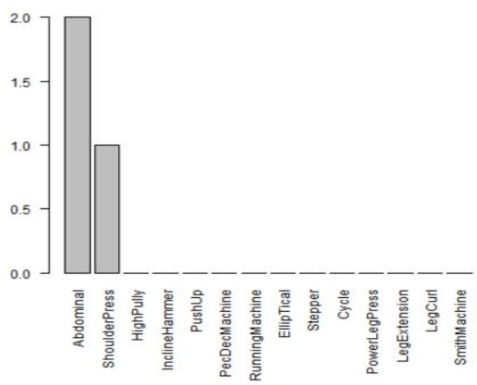

Group Graph Personal Graph

Figure 7. Machine History Graph

\subsection{Review}

For exercise management, users should be provided with information about exercise intensity, exercise amount, and exercise equipment. However, existing exercise simply management systems analyze the height and weight of the user and provide the corresponding information.

However, only individual data of a user is analyzed. This analyzed data is not general information. So this problem must solve. For this problem solve we propose system. The proposed system consists of a analyzes the user's BMI index and BMR value and provides corresponding information. After analyzing the BMI index with the first recommendation algorithm, we recommend exercise intensity. The BMR value is recommended after the second recommendation algorithm is analyzed. And the exercise equipment that the user uses is stored in the database and displayed to the user graphically.

Users can refer to exercise equipment used by other users belonging to the same BMI group as the user, so that the user can compare with the records of exercise equipment he / she has used.

Table 1 shows the comparison between the existing exercise management systems and the proposed system.

Table 1. System Compare and Analysis

\begin{tabular}{ccc}
\hline & Traditional System & Proposed System \\
\hline Body Analysis & Weight based & BMI/BMR Weight \\
Machine Information & Not Offer & Offer 14 Machines \\
Calculation Momentum & Weight based & BMI Index based \\
Recommendation Momentum & insufficient & BMR Value based \\
Reference History & insufficient & Machine History based \\
\hline
\end{tabular}

Existing exercise management systems provided the function of analyzing the user's body weight and calculating the momentum. Body weight-based information cannot accurately analyze the user's body 
information, and if BMR values are not analyzed, the information provided is likely to be inappropriate for the user's body. However the proposed system analyzes the user's BMI index and BMR value based on the information that is studied in U-Healthcare field, and recommends exercise intensity and momentum to the user. Also, since the user selects the exercise device is a fluid aspect, the user can check or modify the exercise record by specifying the exercise device usage log as a graph.

This allows the user to receive various and accurate information compared to existing exercise management systems. So users can exercise or planning of exercise efficient.

\section{CONCLUSION}

The system consists of an Android smartphone application and a Java server for socket database communication and PC communication. Application analyzes the user's BMI index and BMR value and provides information accordingly. The information provided includes exercise intensity and momentum, history of exercise equipment used. In addition, when the user performs the exercise, it detects the movement of the user and calculates the calories consumed by the user in real time. Sends the calories consumed by the database to the user's choice. The database stores user ID, password, body information, BMI group, and recently used exercise equipment information. It also creates a personal table of users and stores the exercise equipment that the user has used you can analyze and refer to it as a graph. Users can refer to the exercise equipment used by users belonging to the same BMI group as the user, so that the user can compare and modify the exercise plan. The system utilizes three recommendation algorithms to provide information, which provides to users various information for exercise. Based on this, it is considered that the user can perform the exercise management more effectively than the existing exercise management system.

\section{ACKNOWLEDGEMENTS}

This research was supported by Basic Science Research Program through the National Research

Foundation of Korea(NRF) funded by the Ministry of Education(No. 2014R1A1A2059842)

This work was supported by the research grant of PaiChai University in 2017.

\section{REFERENCES}

[1] G. T. Ryu, H. Choi, "Implementation of U-Healthcare System for Chronic Disease Management," The Institute of Electronics and Information Engineers, vol. 51, no. 1, pp. 233-240, 2014.

[2] C. M. Kim, G. H. Kang, E. S. Kim, "Active Spinning Training System using Complex Physiological Signals," The Korea Contents Association, vol. 15, no. 7, pp. 591-600, 2015.

[3] M. Sain, W. Y. Chung, H. J. Lee, "A Personalized Healthcare Analysis System in Ubiquitous Environment," Journal of Information and Communication Convergence Engineering, vol. 9, no. 2, pp. 235-243, 2011.

[4] E. J. Lee, H. S. Kim, "Implementation of the Healthcare System Using Smart TV," The Korean Institute of Information Technology, vol. 12, no. 10, pp. 191-199, 2014.

[5] S. W. Kim, S. C. Shim, "Development of Mobile Healthcare System Using ECG Measurement," Journal of the Korea Institute Information and Communication Engineering, vol. 18, no. 8, pp. 2008-2016, 2014.

[6] K. S. Shin, H. J. Jung, S. J. Kwon, S. H. Lee, D. H. Kim, "Development of a Physical Training Management Module Using Smart Devices," Korean Institute of Intelligent Systems, vol. 25, no. 6, pp. 571-577, 2015.

[7] J. W. Lee, "Design of Kalman Filter to Estimate Heart Rate Variability from PPG Signal for Mobile Healthcare," The Korea Institute of Information and Communication Engineering, vol. 8, no. 2, pp. 201-204, 2010.

[8] M. Sain, W. Y. Chung, H. J. Lee, "A Personalized Healthcare Analysis System in Ubiquitous Environment," Journal of Information and Communication Convergence Engineering, vol. 9, no. 2, pp. 235-243, 2011.

\section{BIOGRAPHIES OF AUTHORS}

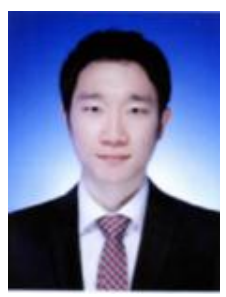

Jongwon Lee. He received the B.S., M.S. degree from the Department of Computer Engineering of Paichai University, Korea in 2014 and 2016. He is currently a Docorate course in Department of Computer Engineering of Paichai University. His current research interests include multimedia information processing, information retrieval system, and semantic web. 


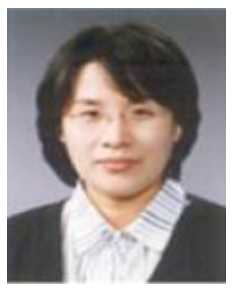

Hyunju Lee. She received the Bachelor of Engineering from the Department of Computer Engineering Education of Andong University, Korea, in 1999, Master degree from the Department of Computer Education of Daejeon University, Korea, in 2003. She is currently a Docorate course in Department of Computer Engineering of Paichai University. She has worked in the Daejeon Girl's Commercial High School, where she now works as a teacher. She current research interests include Data Analysis, Machine Learning.

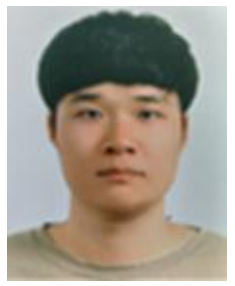

Donggyun Yu. He received the Bachelor of Engineering from the Department of Computer Engineering of Paichai University, Korea, in 2016. He is currently a M. S. degree course in Department of Computer Engineering of Paichai University. His current research interests include Android, IoT and U-Healthcare.

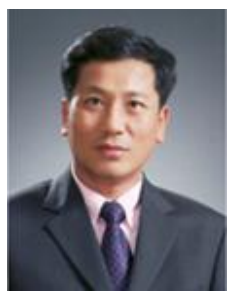

Hoekyung Jung. He received the M.S. degree in 1987 and Ph. D. degree in 1993 from the Department of Computer Engineering of Kwangwoon University, Korea. From 1994 to 1995, he worked for ETRI as a researcher. Since 1994, he has worked in the Department of Computer Engineering at Paichai University, where he now works as a professor. His current research interests include multimedia document architecture modeling, information processing, information retrieval, and databases. 